\title{
Hypocellular Myelodysplastic Syndrome
}

National Cancer Institute

\section{Source}

National Cancer Institute. Hypocellular Myelodysplastic Syndrome. NCI Thesaurus. Code C122686.

Myelodysplastic syndrome characterized by decreased cellularity in the bone marrow. 